\title{
Natriuretic Peptides as an Adjunctive Treatment for Acute Myocardial Infarction
}

\section{Insights from the Meta-analysis of 1,389 Patients from 20 Trials}

\author{
Ting Lyu, ${ }^{1 *}$ MD, Yichao ZhaO, ${ }^{1 *}$ MD, Tuo Zhang, ${ }^{1}$ MD, Wen Zhou, ${ }^{1}$ MD, Fan YANG, ${ }^{1}$ MD, \\ Heng Ge, ${ }^{1} \mathrm{MD}$, Song Ding, ${ }^{1} \mathrm{MD}$, Jun Pu, ${ }^{1} \mathrm{MD}$, and Ben He, ${ }^{1} \mathrm{MD}$
}

\begin{abstract}
SUMMARY
Animal studies have reported a cardioprotective effect for atrial natriuretic peptide (ANP) or brain natriuretic peptide (BNP) administration in the setting of acute myocardial infarction (AMI). However, previous trials performed on AMI patients have reported differences in the cardiac function protection for ANP/BNP infusion, making it difficult to confirm the beneficial effect of natriuretic peptides. We performed a meta-analysis of all available trials to determine whether ANP/BNP infusion was effective in improving cardiac function. Methods: We searched various databases including PubMed, EMBASE, Web of Knowledge, the Cochrane Library, and CKNI for studies related to ANP/BNP infusion in AMI. The major outcome was left ventricular ejection fraction (LVEF). Results: Twenty trials (4 for ANP, 16 for BNP) with 1389 patients were included. There were no significant differences in patient characteristics between the ANP/BNP infusion and control groups at baseline. Pooled analysis showed that patients in the ANP/BNP infusion group had significantly higher LVEF than in the control during follow-up [Studies on ANP: weighted mean differences (WMD) $2.94 \%, 95 \%$ confidence interval (CI): $1.39 \%-4.50 \%, P=0.0002$; Studies on BNP: WMD 4.45\%, 95\%CI: $2.25 \%-6.65 \%$, $P<0.0001]$. Conclusions: The present meta-analysis suggested that ANP/BNP infusion might be effective in protecting left ventricular function in patients with AMI. ANP/BNP infusion may be an effective adjunctive therapy for cardiac function protection in AMI patients. (Int Heart J 2014; 55: 8-16)
\end{abstract}

Key words: Atrial natriuretic peptide, Brain natriuretic peptide, Left ventricular function

$\mathrm{A}$ lthough primary percutaneous coronary intervention (PPCI) or thrombolysis therapy could reestablish normal epicardial blood flow in most cases after onset of acute myocardial infarction (AMI), myocardial damage is still usually unavoidable and may result in heart failure caused by adverse left ventricular remodeling. Under these circumstances, effective adjunct therapies are necessary to improve the prognosis of AMI. Atrial natriuretic peptide (ANP) and brain natriuretic peptide (BNP) have been approved to treat heart failure. ${ }^{1,2)}$ They share similar biological actions such as suppressing the renin-angiotensin-aldosterone system and sympathetic system. ${ }^{3)}$ Previous experimental studies revealed that natriuretic peptide infusion could limit myocardial infarct size and prevent reperfusion arrhythmia in myocardial ischemia-reperfusion injured rats. ${ }^{4-6)}$ Thereafter, several trials were conducted to determine the beneficial role of ANP/BNP infusion on AMI; however, the results were conflicting. In order to provide a more robust evaluation of the potential benefits of ANP/BNP infusion in AMI patients, we performed a systematic review and meta-analysis.

\begin{abstract}
MethodS
Search strategy: We searched the databases such as PubMed, EMBASE, Web of Knowledge, the Cochrane Library, and China National Knowledge Infrastructure (CKNI) (all from their inception to March 2013). The search terms were as follows: natriuretic peptide, cardiac peptide, atrial natriuretic peptide, atrium natriuretic peptide, carperitide, brain natriuretic peptide, B-type natriuretic peptide, nesiritide, acute myocardial infarction, acute heart infarction, ST-segment elevated myocardial infarction, ST-segment elevation myocardial infarction, non-ST-segment elevated myocardial infarction, non-ST-segment elevation myocardial infarction, therapy, treatment, and treat. Various combinations of these terms were used depending on the requirements of the databases. No language restriction was used.

Study selection: Inclusion criteria for retrieved studies were: (1) randomized controlled trials (RCTs) based on AMI routine therapy (eg, aspirin, clopidogrel, angiotensin-converting enzyme inhibitors, $\beta$-blockers, and statins) comparing ANP or
\end{abstract}

From the ${ }^{1}$ Department of Cardiology, Renji Hospital, School of Medicine, Shanghai Jiaotong University, Shanghai, China.

* These authors contributed equally to this work.

This work was supported by the International Cooperation Program of Shanghai Committee of Science and Technology (12410708300), Program of Shanghai Hospital Development Center (SHDC12013119), Program for New Century Excellent Talents in University (NCET-12-0352), and Shanghai Shuguang Program (12SG22).

Address for correspondences: Jun Pu, MD, or Ben He, MD, Department of Cardiology Renji Hospital School of Medicine Shanghai Jiaotong University, Pu Jian Rd

No.160, Shanghai 200127, China. E-mail: heben1026@hotmail.com

Received for publication May 2, 2013.

Revised and accepted August 8, 2013.

Released advance online J-STAGE January 27, 2014.

All rights are reserved to the International Heart Journal Association. 
BNP infusion with control groups in patients with AMI; (2) diagnosis of AMI: ST-segment elevated myocardial infarction (STEMI) was defined as typical clinical presentation, ST-segment elevation $(>0.2 \mathrm{mV}$ elevation in two or more adjacent precordial leads, or $>0.1 \mathrm{mV}$ in adjacent limb leads) and a rise in plasma cardiac markers; non-ST-segment elevated myocardial infarction was defined as typical clinical presentation and elevation of plasma cardiac markers, with no ST-segment elevation on ECG; (3) data on myocardial infarction size, left ventricular ejection fraction (LVEF), or major adverse cardiovascular events (MACEs). Studies without reports of relevant clinical outcomes were excluded.

Data extraction and quality assessment: Data abstraction was independently performed by two unblinded reviewers. Discrepancies were resolved by consensus. Methodological quality was evaluated using the Jadad score, which assigned 0 or 1 points to each of the following 5 items: (i) with or without randomization; (ii) with or without a double-blind design; (iii) the appropriateness of the randomization methods if used; (iv) the appropriateness of double-blinding design if used; and (v) the analysis and reasons for withdrawals and dropouts. ${ }^{7}$ Thus, the Jadad scores could range from 0 to 5 . The quality of studies to be included was assessed independently by two reviewers. Studies would be deemed as excellent quality if they received a Jadad score of 5 (of a possible 5 points), good quality if the score was 3 or 4 , and poor quality if the score was $\leq 2$.

Data synthesis and data analysis: Pooled effects were presented as weighted mean differences (WMD) or odds ratios (ORs) with $95 \%$ confidence intervals (CI) using random effects models. Statistical heterogeneity was measured using the $\mathrm{I}^{2}$ statistic $\left(\mathrm{I}^{2}>50 \%\right.$ was considered representative of significant statistical heterogeneity). Meta-regression was conducted if there was significant heterogeneity. Finally, on the basis of the data on LVEF, publication bias was tested using the Begg adjustedrank correlation test and Egger regression asymmetry test. $P$ values were 2-tailed, and the statistical significance was set at 0.05. All analyses were carried out with Rev-Man Analyses Version 5.0 or Stata software 12.0 (StataCorp, College Station, TX).

\section{RESULTS}

Search results and study selection: The flow of selection of studies for inclusion in the meta-analysis is shown in Figure 1. Database searches retrieved an initial total of 3090 articles. These included editorials, reviews, and letters. Through screening of abstracts, 52 articles were deemed relevant; of which we went through full-text screening. Finally, 20 RCTs meeting inclusion/exclusion criteria were retained in the analysis, of which 4 were ANP related, and 16 were BNP related. ${ }^{8-27)}$ These studies involved 1389 patients: 229 patients in the ANP groups (272 patients as controls); and 442 patients in the BNP groups (446 patients as controls).

Studies of ANP: The general characteristics of the collected studies of ANP are described in Table I. All the studies were prospective and RCTs. The patients included were all STEMI, of which 3 were first anterior STEMI. LVEF was evaluated through left ventriculography. In the study by Kitakaze, et al, LVEF was measured twice during the follow-up (2-8 weeks with median time 18 days, and 6-12 months with median time

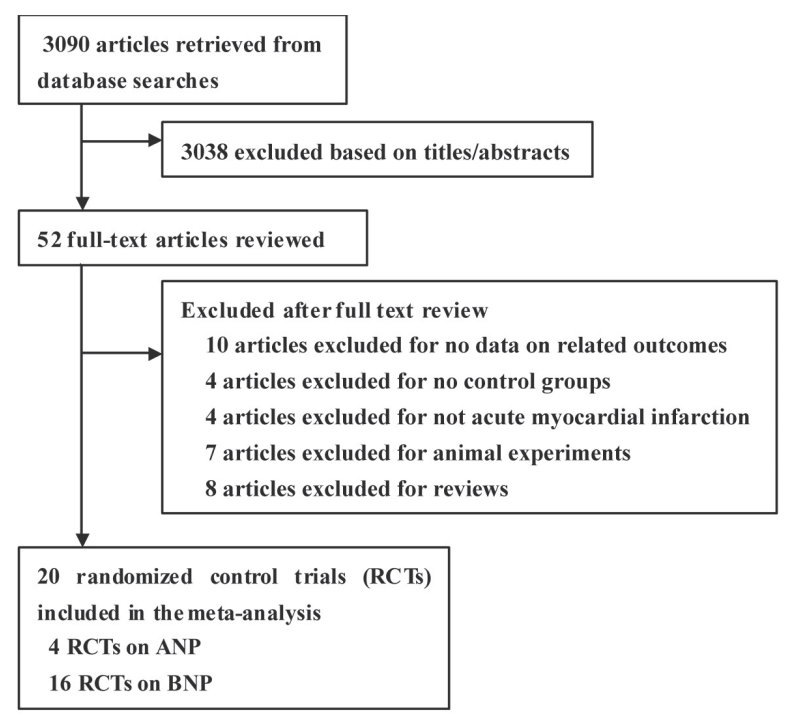

Figure 1. Review Process.

Table I. General Characteristics of Studies on Atrial Natriuretic Peptide (ANP)

\begin{tabular}{ccccccccccc}
\hline Study & $\begin{array}{c}\text { Publication } \\
\text { Year }\end{array}$ & Location & $\begin{array}{c}\text { Total } \\
\text { patients }\end{array}$ & AHF & $\begin{array}{c}\text { Control } \\
\text { Group }\end{array}$ & $\begin{array}{c}\text { Duration } \\
\text { (hour) }\end{array}$ & $\begin{array}{c}\text { LVEF } \\
\text { Measurement } \\
\text { Method }\end{array}$ & $\begin{array}{c}\text { LVEF } \\
\text { Measurement } \\
\text { Time }\end{array}$ & $\begin{array}{c}\text { Follow-up } \\
\text { Time }\end{array}$ & $\begin{array}{c}\text { Jadad } \\
\text { Score }\end{array}$ \\
\hline $\begin{array}{c}\text { Hayashi } \\
\text { et al }\end{array}$ & 2001 & Japan & 60 & NO & GTN & $61.5 \pm 30.7$ & $\begin{array}{c}\text { Left } \\
\text { ventriculography } \\
\text { Left }\end{array}$ & 1 months & 1 month & 3 \\
$\begin{array}{c}\text { Kuga } \\
\text { et al } \\
\text { Kasama } \\
\text { et al }\end{array}$ & 2003 & Japan & 37 & NO & $\begin{array}{c}\text { Physiological } \\
\text { Saline }\end{array}$ & 168 & $\begin{array}{c}\text { ventriculography } \\
\text { Left }\end{array}$ & 6 months & 6 months & 3 \\
$\begin{array}{c}\text { Kitakaze } \\
\text { et al }\end{array}$ & 2007 & Japan & 50 & NO & ISDN & $56 \pm 15$ & $\begin{array}{c}2 \text { weeks } \\
\text { ventriculography }\end{array}$ & $\begin{array}{c}2 \text { 2-8 weeks } \\
\text { Left } \\
\text { (median 18days), } \\
\text { 6-12 months }\end{array}$ & 2.7 years \\
(median 196days)
\end{tabular}

NA indicates not available; AHF, acute heart failure; LVEF, left ventricular ejection fraction; GTN, nitroglycerin; GS, glucose solution; and ISDN, isosorbide dinitrate. 
Table II. Basic Characteristics of Studies on Atrial Natriuretic Peptide (ANP)

\begin{tabular}{|c|c|c|c|c|c|c|c|c|c|}
\hline Study & Groups & $n$ & Age & Male & PCI & ANP dose & $\begin{array}{c}\text { Primary } \\
\operatorname{LVEF}(\%)\end{array}$ & $\begin{array}{l}\text { Renal } \\
\text { failure }\end{array}$ & Hypotension \\
\hline \multirow[t]{2}{*}{$\begin{array}{l}\text { Hayashi } \\
\text { et al }\end{array}$} & ANP & 30 & $60.9 \pm 10.4$ & 19 & $100 \%$ & $0.028 \pm 0.002 \mu \mathrm{g} / \mathrm{kg} /$ minute & $46.9 \pm 5.5$ & NA & NA \\
\hline & Control & 30 & $59.4 \pm 11.5$ & 21 & $100 \%$ & $\begin{array}{c}0.476 \pm 0.04 \mu \mathrm{g} / \mathrm{kg} / \text { minute } \\
0.025 \mu \mathrm{g} / \mathrm{kg} / \mathrm{minute}\end{array}$ & $46.8 \pm 7.1$ & NA & NA \\
\hline \multirow[t]{2}{*}{$\begin{array}{l}\text { Kuga } \\
\text { et al }\end{array}$} & ANP & 19 & $60.1 \pm 11$ & 15 & $100 \%$ & $\begin{array}{c}\text { intravenous } \\
\text { injection }+25 \mathrm{mg} \\
\text { intracoronary injection }\end{array}$ & $53.1 \pm 12.3$ & NA & NA \\
\hline & Control & 18 & $58.7 \pm 9$ & 13 & $100 \%$ & $\begin{array}{l}\text { The same volume as the } \\
\text { ANP group }\end{array}$ & $52.2 \pm 14.2$ & NA & NA \\
\hline \multirow{2}{*}{$\begin{array}{c}\text { Kasama } \\
\text { et al }\end{array}$} & ANP & 25 & $61 \pm 12$ & 20 & $100 \%$ & $0.025 \mu \mathrm{g} / \mathrm{kg} / \mathrm{minute}$ & NA & NA & NA \\
\hline & Control & 25 & $62 \pm 13$ & 19 & $100 \%$ & $0.67 \mu \mathrm{g} / \mathrm{kg} / \mathrm{minute}$ & NA & NA & NA \\
\hline \multirow{2}{*}{$\begin{array}{l}\text { Kitakaze } \\
\text { et al }\end{array}$} & ANP & 155 & $63.0 \pm 10.4$ & 118 & $100 \%$ & $0.025 \mu \mathrm{g} / \mathrm{kg} / \mathrm{minute}$ & NA & 0 & 29 \\
\hline & Control & 199 & $61.8 \pm 10.7$ & 166 & $100 \%$ & $\begin{array}{l}\text { The same volume as the } \\
\text { ANP group }\end{array}$ & NA & 0 & 1 \\
\hline
\end{tabular}

LVEF indicates left ventricular ejection fraction; NA, not available; and PCI, percutaneous coronary intervention.

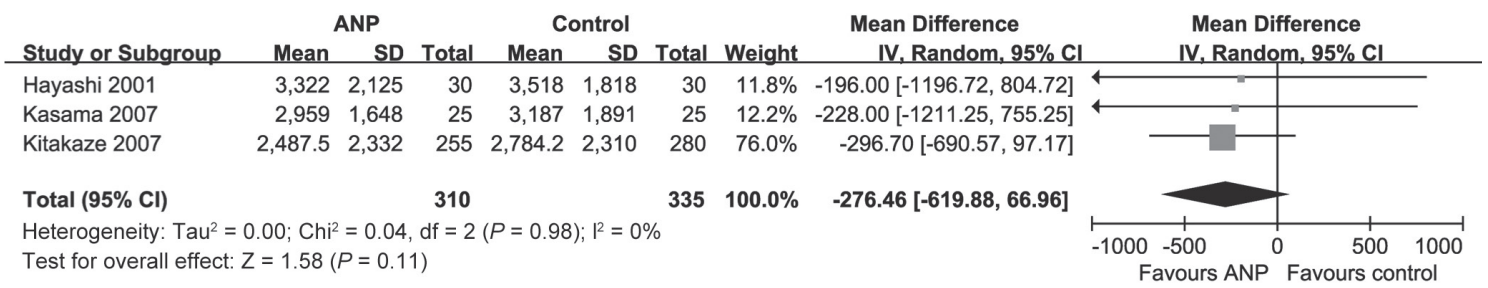

Figure 2. Creatine kinase (CK) peak comparison between atrial natriuretic peptide (ANP) group and control group.

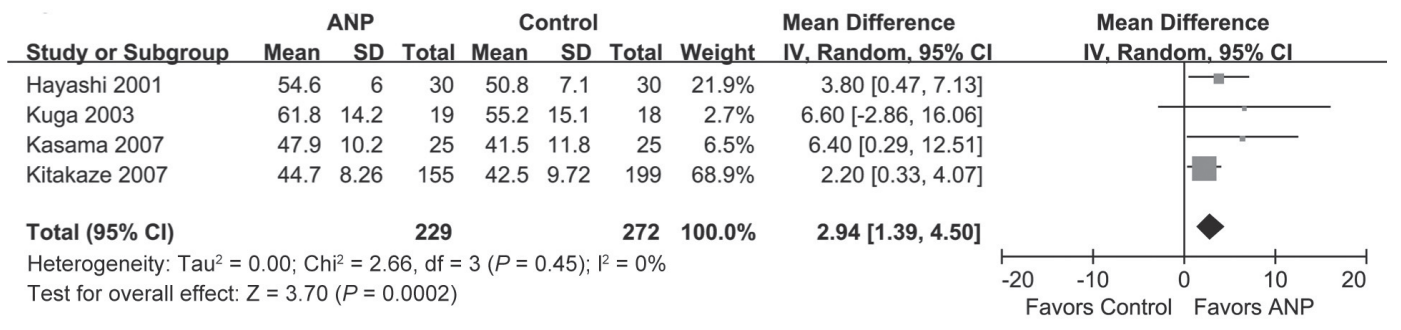

Figure 3. Left ventricular ejection fraction (LVEF) comparison between atrial natriuretic peptide (ANP) group and control group at follow-up.

196 days). ${ }^{16)}$ The mean follow-up time was 2 weeks to 2.7 years. No patient in the 4 trials was complicated with acute heart failure (AHF). Table II lists the basic characteristics of the studies, from which no significant differences were observed between the subjects in the ANP group and the control group. The reported mean age of the patients ranged from 60.1 to 63.0 years, and each trial enrolled mostly men. All patients in the 4 studies received PPCI; and patients received ANP infusion only when their hemodynamic states were stable. Three studies gave the patients ANP intravenously, and one study both intravenously and via intracoronary administration. The mean infusion period was 56 to 168 hours.

We compared the creatine kinase $(\mathrm{CK})$ peak between the ANP infusion group and the control group. The pooled result showed that no obvious difference was observed between the two groups [WMD -276.46, 95\%CI: (-619.88)-66.96, $\mathrm{I}^{2}=0 \%$ ]
(Figure 2). The overall pooled results with random effects analysis showed that additional ANP treatment was significantly superior to standard medical therapy in terms of LVEF improvement (WMD 2.94\%, 95\% CI: $1.39 \%-4.50 \%, P=$ 0.0002) (Figure 3 ). In addition, considering the LVEF measurement time varied from 2 weeks to a median time of 196 days, we performed a subgroup analysis of studies with shortterm (within 3 months) and relative long-term (over 3 months) LVEF data. The results indicated that LVEF was improved compared with the control group both at the short-term followup (WMD 2.87\%, 95\% CI: $0.67 \%-5.06 \%$ ) and the relative long-term follow-up (WMD 2.37\%, 95\%CI: 0.53\%-4.21\%). Furthermore, subgroup analysis of ANP studies with different infusion durations was performed. The results showed that LVEF was increased by $4.39 \%$ in the ANP group with an infusion time less than 72 hours compared with the control group 
Table III. General Characteristics of Studies on Brain Natriuretic Peptide (BNP)

\begin{tabular}{|c|c|c|c|c|c|c|c|c|c|c|}
\hline Study & $\begin{array}{c}\text { Publication } \\
\text { Year }\end{array}$ & Location & $\begin{array}{c}\text { Total } \\
\text { patients }\end{array}$ & $\mathrm{AHF}$ & Control Group & $\begin{array}{l}\text { Duration } \\
\text { (hour) }\end{array}$ & $\begin{array}{c}\text { LVEF } \\
\text { Measurement } \\
\text { Method }\end{array}$ & $\begin{array}{c}\text { LVEF } \\
\text { Measurement } \\
\text { Time }\end{array}$ & $\begin{array}{l}\text { Follow-up } \\
\text { Time }\end{array}$ & $\begin{array}{l}\text { Jadad } \\
\text { Score }\end{array}$ \\
\hline $\begin{array}{l}\text { Cai } \\
\text { et al }\end{array}$ & 2010 & China & 46 & YES & GTN & 72 & Echocardiography & NA & NA & 3 \\
\hline $\begin{array}{l}\text { Chen } \\
\text { et al }\end{array}$ & 2009 & U.S. & 24 & NO & Low dose BNP & 24 & $\begin{array}{l}\text { Gated equilibrium } \\
\text { radionuclide } \\
\text { ventriculography }\end{array}$ & 1 month & 1 month & 2 \\
\hline $\begin{array}{l}\text { Chen } \\
\text { et al }\end{array}$ & 2011 & China & 82 & NO & Blank & 72 & Echocardiography & $\begin{array}{l}12 \text { weeks, } \\
24 \text { weeks }\end{array}$ & 24 weeks & 3 \\
\hline $\begin{array}{l}\text { Ding } \\
\text { et al }\end{array}$ & 2010 & China & 30 & NO & $\begin{array}{l}\text { Physiological } \\
\text { saline }\end{array}$ & $24-48$ & Echocardiography & 3 months & 3 months & 2 \\
\hline $\begin{array}{l}\text { Duan } \\
\text { et al }\end{array}$ & 2007 & China & 24 & NO & Blank & 30 & Echocardiography & 6 months & 6 months & 3 \\
\hline $\begin{array}{l}\text { Hillock } \\
\text { et al }\end{array}$ & 2007 & $\begin{array}{c}\text { New } \\
\text { Zealand }\end{array}$ & 28 & NO & $\begin{array}{l}\text { Physiological } \\
\text { saline }\end{array}$ & 60 & Echocardiography & 3 months & 3 months & 4 \\
\hline $\begin{array}{c}\mathrm{Li} \\
\text { et al }\end{array}$ & 2011 & China & 52 & NO & Blank & 72 & Echocardiography & $\begin{array}{l}\text { 3months, } \\
6 \text { months to } \\
1 \text { year }\end{array}$ & $\begin{array}{c}6 \text { months to } \\
1 \text { year }\end{array}$ & 2 \\
\hline $\begin{array}{l}\text { Liu } \\
\text { et al }\end{array}$ & 2010 & China & 70 & YES & GTN & 72 & Echocardiography & 3 months & 3 months & 3 \\
\hline $\begin{array}{l}\text { Liu } \\
\text { et al }\end{array}$ & 2011 & China & 66 & YES & GTN & 72 & Echocardiography & 3days & 3days & 3 \\
\hline $\begin{array}{l}\text { Long } \\
\text { et al }\end{array}$ & 2011 & China & 64 & YES & Blank & 48 & Echocardiography & NA & NA & 3 \\
\hline $\begin{array}{l}\mathrm{Ma} \\
\text { et al }\end{array}$ & 2009 & China & 100 & YES & Blank & $72-120$ & Echocardiography & 1 month & 1 month & 3 \\
\hline $\begin{array}{l}\text { Wang } \\
\text { et al }\end{array}$ & 2008 & China & 86 & YES & ISDN & $\mathrm{NA}^{*}$ & Echocardiography & 6 months & 6 months & 4 \\
\hline $\begin{array}{l}\text { Xue } \\
\text { et al }\end{array}$ & 2009 & China & 48 & NO & Blank & 72 & Echocardiography & $\begin{array}{l}4 \text { weeks, } \\
24 \text { weeks }\end{array}$ & 24 weeks & 3 \\
\hline $\begin{array}{l}\text { Zhang } \\
\text { et al }\end{array}$ & 2010 & China & 87 & YES & GTN & 24 & Echocardiography & NA & NA & 3 \\
\hline $\begin{array}{l}\text { Zhao } \\
\text { et al }\end{array}$ & 2010 & China & 60 & NO & $\begin{array}{c}\text { Sodium } \\
\text { nitroprusside }\end{array}$ & 72 & Echocardiography & 3 months & 3 months & 3 \\
\hline $\begin{array}{l}\text { Zou } \\
\text { et al }\end{array}$ & 2007 & China & 30 & NO & Blank & 24 & Echocardiography & NA & NA & 3 \\
\hline
\end{tabular}

*Duration time to a total BNP dose of $2 \mathrm{mg}$; NA indicates not available, AHF, acute heart failure, LVEF, left ventricular ejection fraction; GTN, nitroglycerin; and ISDN, isosorbide dinitrate. Blank means that patients in the control group did not receive extra intravenous injections of any drugs other than common therapy for AMI.

(95\%CI: $1.47 \%-7.32 \%$ ), and $2.37 \%$ in the ANP group with an infusion time over 72 hours (95\%CI: $0.53 \%-4.21 \%$ ).

Of the 4 ANP studies, only the study by Kitakaze, et al had data on MACE. ${ }^{16)}$ In that study, although no significant differences were observed between the ANP group and control group in either survival rates (Hazard ratio $=0.693,95 \% \mathrm{CI}$ : $0.269-1.788, P=0.446$ ) or the incidence of cardiovascular events (Hazard ratio $=0.833,95 \%$ CI: $0.608-1.140, P=0.252$ ), the rates of cardiac death and re-admission to hospital for heart failure were both lower in patients given ANP than in controls (Hazard Ratio $=0.267,95 \%$ CI: $0.089-0.799, P=0.0112)$ at median follow-up of 2.7 years. The study by Kasama, et al reported that during reperfusion, the incidence of additional electrocardiographic ST-segment elevation in the ANP group was significantly lower than in the control group (20\% versus $35 \%$; $P<0.05)$, and that of reperfusion arrhythmias was also lower in the ANP group compared with the control group (28\% versus $48 \%$; $P<0.05) .{ }^{15)}$ Of the 4 studies, only that by Kitakaze mentioned incidences of hypotension and renal function deterioration during ANP infusion, which were the main side effects of this drug. The patients in the ANP group showed no obvious changes in renal function compared with the basal state, but 29 patients developed hypotension as compared with only one in the control group. ${ }^{16}$

Studies of BNP: The general characteristics of the 16 BNP studies are described in Table III. The studies were performed from 2006 to 2010. LVEF were mostly evaluated through echocardiography. Four studies did not mention follow-up duration. Basic information on the studies is shown in Table IV. One study did not provide the basic characteristics of the patients, but mentioned that there were no obvious differences between the BNP group and control group. ${ }^{22)}$ Seven studies included AMI patients complicated with acute heart failure. All regimens were given intravenously.

The pooled results showed that compared with the control group, BNP therapy did not reduce infarct size as estimated by CK-MB [WMD -20.19, 95\%CI: (-68.78)-28.4, $\mathrm{I}^{2}=$ $62 \%$ ] (Figure 4). Pooled analysis with a random-effects model showed an improvement of LVEF in the BNP group compared with the control during follow-up (WMD 4.45\%, 95\%CI: $2.25 \%-6.65 \%, P<0.0001, \mathrm{I}^{2}=89 \%$ ) (Figure 5). We further performed a subgroup analysis of BNP studies with short-term (within 3 months) and relative long-term (over 3 months) LVEF data. The results showed that LVEF was improved com- 
Table IV. Basic Characteristics of Studies on Brain Natriuretic Peptide (BNP)

\begin{tabular}{|c|c|c|c|c|c|c|c|c|c|}
\hline Study & Groups & $n$ & Age & Male & PCI & ANP dose & $\begin{array}{c}\text { Primary } \\
\operatorname{LVEF}(\%)\end{array}$ & Renal failure & Hypotension \\
\hline \multirow[t]{2}{*}{$\begin{array}{l}\text { Cai } \\
\text { et al }\end{array}$} & BNP & 22 & $69.3 \pm 7.8$ & 13 & NA & $\begin{array}{l}\text { Loading dose: } 1.5-2.0 \mu \mathrm{g} / \mathrm{kg} \\
\text { then } 0.0075-0.01 \mu \mathrm{g} / \mathrm{kg} / \text { minute }\end{array}$ & $28 \pm 32$ & 0 & 2 \\
\hline & Control & 24 & $66.4 \pm 8.4$ & 14 & NA & $\begin{array}{c}5-10 \mu \mathrm{g} / \text { minute; gradually } \\
\text { titrated up }\end{array}$ & $29 \pm 31$ & 0 & 7 \\
\hline \multirow{2}{*}{$\begin{array}{l}\text { Chen } \\
\text { et al }\end{array}$} & BNP & 12 & $58 \pm 4$ & 9 & $100 \%$ & $0.006 \mu \mathrm{g} / \mathrm{kg} / \mathrm{minute}$ & $42 \pm 2$ & 0 & 2 \\
\hline & Control & 12 & $59 \pm 3$ & 6 & $91.7 \%$ & $0.003 \mu \mathrm{g} / \mathrm{kg} /$ minute & $40 \pm 4$ & 0 & 2 \\
\hline \multirow{2}{*}{$\begin{array}{l}\text { Chen } \\
\text { et al }\end{array}$} & BNP & 24 & $65 \pm 12.1$ & 29 & $100 \%$ PPCI & $\begin{array}{l}\text { Loading dose: } 1.5 \mu \mathrm{g} / \mathrm{kg} \\
\text { then } 0.0075 \mu \mathrm{g} / \mathrm{kg} / \text { minute }\end{array}$ & NA & NA & NA \\
\hline & Control & 28 & $58 \pm 15.8$ & 28 & $100 \%$ PPCI & - & NA & NA & NA \\
\hline \multirow[t]{2}{*}{$\begin{array}{l}\text { Ding } \\
\text { et al }\end{array}$} & $\mathrm{BNP}$ & 15 & $55.2 \pm 8.7$ & 13 & $100 \%$ PPCI & $\begin{array}{l}\text { Loading dose: } 1.5 \mu \mathrm{g} / \mathrm{kg} \\
\text { then } 0.0075 \mu \mathrm{g} / \mathrm{kg} / \text { minute }\end{array}$ & $52.9 \pm 5.0$ & NA & 4 \\
\hline & Control & 15 & $59.9 \pm 13.6$ & 11 & $100 \%$ PPCI & the same dose & $51.4 \pm 6.4$ & NA & NA \\
\hline \multirow[t]{2}{*}{$\begin{array}{l}\text { Duan } \\
\text { et al }\end{array}$} & $\mathrm{BNP}$ & 12 & $61.4 \pm 6.5$ & 11 & $100 \%$ PPCI & $\begin{array}{l}\text { Loading dose: } 1.5 \mu \mathrm{g} / \mathrm{kg} \text {; } \\
\text { then } 0.0075 \mu \mathrm{g} / \mathrm{kg} / \text { minute }\end{array}$ & $48.83 \pm 2.98$ & 0 & NA \\
\hline & Control & 12 & $60.5 \pm 5.4$ & 10 & $100 \%$ PPCI & - & $44.5 \pm 4.6$ & 0 & NA \\
\hline \multirow{2}{*}{$\begin{array}{l}\text { Hillock } \\
\text { et al }\end{array}$} & BNP & 14 & $62.4 \pm 2.3$ & 12 & $91 \%$ & $0.01 \mu \mathrm{g} / \mathrm{kg} /$ minute & $42.1 \pm 1.8$ & 0 & 0 \\
\hline & Control & 13 & $67.9 \pm 3.4$ & 10 & $92 \%$ & The same volume & $43.6 \pm 1.4$ & 0 & 0 \\
\hline \multirow{2}{*}{$\begin{array}{l}\mathrm{Li} \\
\text { et al }\end{array}$} & BNP & 24 & $65 \pm 12.1$ & NA & $100 \%$ & $\begin{array}{l}\text { Loading dose: } 1.5 \mu \mathrm{g} / \mathrm{kg} \\
\text { then } 0.0075 \mu \mathrm{g} / \mathrm{kg} / \mathrm{minute}\end{array}$ & NA & NA & NA \\
\hline & Control & 28 & $58 \pm 15.8$ & NA & $100 \%$ & - & NA & NA & NA \\
\hline \multirow[t]{2}{*}{$\begin{array}{l}\text { Liu } \\
\text { et al }\end{array}$} & BNP & 35 & $62 \pm 10.5$ & 18 & $44.4 \%$ & $\begin{array}{l}\text { Loading dose: } 1.5 \mu \mathrm{g} / \mathrm{kg} \text {; } \\
\text { then } 0.0075 \mu \mathrm{g} / \mathrm{kg} / \text { minute }\end{array}$ & $40.52 \pm 2.35$ & NA & NA \\
\hline & Control & 35 & $63 \pm 11.2$ & 19 & $36.8 \%$ & Loading dose: $1.5 \mu \mathrm{g} / \mathrm{kg}$ & $39.47 \pm 1.23$ & NA & NA \\
\hline \multirow[t]{2}{*}{$\begin{array}{l}\text { Liu } \\
\text { et al }\end{array}$} & BNP & 33 & $67.5 \pm 11.2$ & 19 & none & $\begin{array}{c}\text { then } 0.0075 \mu \mathrm{g} / \mathrm{kg} / \text { minute for } \\
3 \mathrm{~h} \text {, finally } 0.015-0.03 \\
\mu \mathrm{g} / \mathrm{kg} / \text { minute }\end{array}$ & $47 \pm 9$ & NA & NA \\
\hline & Control & 33 & $63.6 \pm 12.8$ & 17 & none & $\begin{array}{l}10 \mu \mathrm{g} / \text { minute; gradually } \\
\text { titrated up }\end{array}$ & $46 \pm 8$ & NA & NA \\
\hline \multirow[t]{2}{*}{$\begin{array}{l}\text { Long } \\
\text { et al }\end{array}$} & BNP & 32 & $64.1 \pm 12.1$ & 18 & NA & $\begin{array}{l}\text { Loading dose: } 1.5 \mu \mathrm{g} / \mathrm{kg} \\
\text { then } 0.0075 \mu \mathrm{g} / \mathrm{kg} / \text { minute }\end{array}$ & $48 \pm 9$ & 0 & 1 \\
\hline & Control & 32 & $63.0 \pm 11.3$ & 17 & NA & - & $48 \pm 11$ & 0 & 0 \\
\hline \multirow{2}{*}{$\begin{array}{l}\text { Ma } \\
\text { et al }\end{array}$} & BNP & 50 & NA & NA & NA & NA & NA & NA & NA \\
\hline & Control & 50 & NA & NA & NA & NA & NA & NA & NA \\
\hline \multirow[t]{2}{*}{$\begin{array}{l}\text { Wang } \\
\text { et al }\end{array}$} & $\mathrm{BNP}$ & 43 & $69.8 \pm 11.3$ & 28 & $65.1 \%$ & $\begin{array}{l}0.015-0.03 \mu \mathrm{g} / \mathrm{kg} / \mathrm{minute}, \\
\text { total dose } 2 \mathrm{mg}\end{array}$ & $38.7 \pm 13.3$ & 0 & 1 \\
\hline & Control & 43 & $70.3 \pm 13.7$ & 29 & $67.4 \%$ & $\begin{array}{l}\text { titrated up according to } \\
\text { the pressure }\end{array}$ & $39.4 \pm 12.2$ & 0 & 7 \\
\hline \multirow[t]{2}{*}{$\begin{array}{l}\text { Xue } \\
\text { et al }\end{array}$} & $\mathrm{BNP}$ & 25 & $55.7 \pm 5.8$ & 17 & $100 \%$ PPCI & $\begin{array}{l}\text { Loading dose: } 1.5 \mu \mathrm{g} / \mathrm{kg} \text {; } \\
\text { then } 0.0075 \mu \mathrm{g} / \mathrm{kg} / \text { minute }\end{array}$ & NA & 0 & 0 \\
\hline & Control & 23 & $58.9 \pm 6.1$ & 16 & $100 \%$ PPCI & Loading dose: $1.5 \mu \mathrm{g} / \mathrm{kg}$ & NA & 0 & 0 \\
\hline \multirow[t]{2}{*}{$\begin{array}{l}\text { Zhang } \\
\text { et al }\end{array}$} & BNP & 43 & $68.0 \pm 5.7$ & 34 & $83.70 \%$ & $\begin{array}{c}\text { then } 0.0075 \mu \mathrm{g} / \mathrm{kg} / \text { minute; } \\
\text { titrated up to } 0.015-0.03 \\
\mu \mathrm{g} / \mathrm{kg} / \text { minute }\end{array}$ & $36.42 \pm 5.84$ & 0 & NA \\
\hline & Control & 44 & $68.5 \pm 6.0$ & 32 & $79.50 \%$ & $\begin{array}{l}20-100 \mu \mathrm{g} / \text { minute; gradually } \\
\text { titrated up }\end{array}$ & $35.86 \pm 6.22$ & 0 & NA \\
\hline $\begin{array}{l}\text { Zhao } \\
\text { et al }\end{array}$ & $\mathrm{BNP}$ & 27 & $67.8 \pm 7.0$ & 14 & 0 & $\begin{array}{l}\text { Loading dose: } 0.5 \mu \mathrm{g} / \mathrm{kg} \\
\text { in } 90 \mathrm{~s} ; \text { then } 0.0075 \\
\mu \mathrm{g} / \mathrm{kg} / \mathrm{minute} ; \text { titrated up to } \\
0.015-0.03 \mu \mathrm{g} / \mathrm{kg} / \text { minute }\end{array}$ & $33.9 \pm 4.6$ & 0 & 0 \\
\hline & Control & 28 & $66.4 \pm 9.3$ & 14 & 0 & $\begin{array}{l}\text { Initial rate: } 10 \mu \mathrm{g} / \text { minute; } \\
\text { gradually titrated up }\end{array}$ & $36.4 \pm 4.8$ & 3 & 0 \\
\hline \multirow[t]{2}{*}{$\begin{array}{l}\text { Zou } \\
\text { et al }\end{array}$} & $\mathrm{BNP}$ & 15 & $68 \pm 11$ & 10 & NA & $\begin{array}{l}\text { Loading dose: } 1.5 \mu \mathrm{g} / \mathrm{kg} \text {; } \\
\text { then } 0.0075 \mu \mathrm{g} / \mathrm{kg} / \text { minute }\end{array}$ & $35 \pm 10$ & 0 & 1 \\
\hline & Control & 15 & $67 \pm 9$ & 9 & NA & - & $36 \pm 10$ & 0 & 0 \\
\hline
\end{tabular}

LVEF indicates left ventricular ejection fraction; NA, not available; PCI, percutaneous coronary intervention; and PPCI, primary percutaneous coronary intervention. 


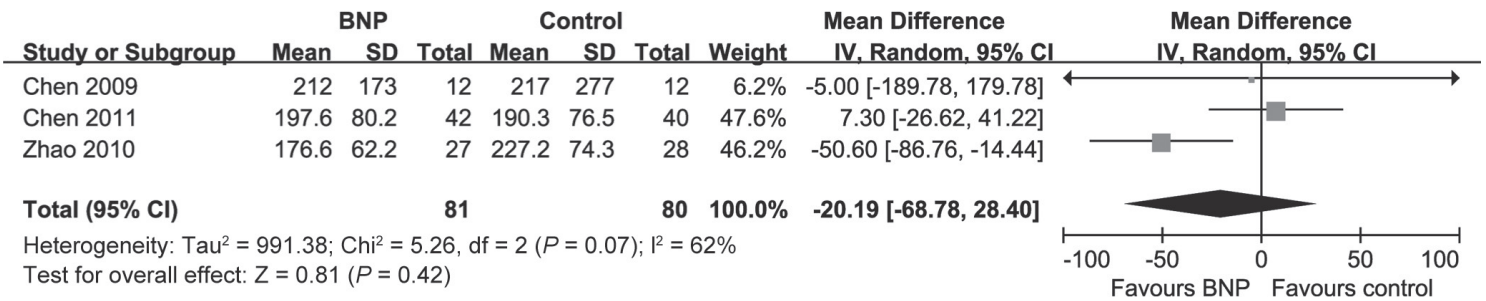

Figure 4. Creatine kinase (CK)-MB peak comparison between brain natriuretic peptide (BNP) group and control group.

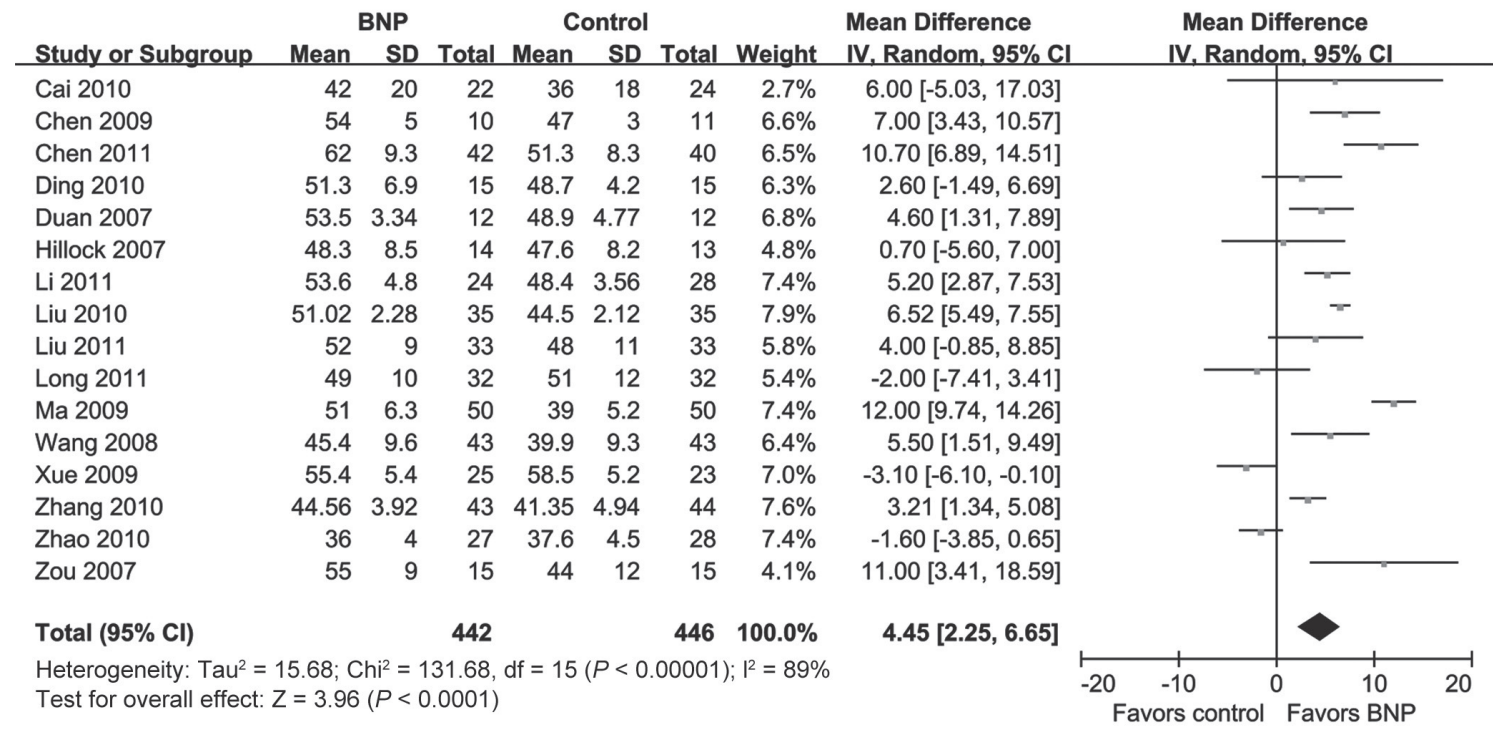

Figure 5. Left ventricular ejection fraction (LVEF) comparison between group administered brain natriuretic peptide (BNP) and the control group at follow-up.

\begin{tabular}{|c|c|c|c|c|c|c|c|c|c|c|}
\hline Study or Subgroup & $\begin{array}{l}\text { BNP } \\
\text { Events }\end{array}$ & Total & $\begin{array}{l}\text { Contr } \\
\text { Events }\end{array}$ & & Weight & $\begin{array}{c}\text { Odds Ratio } \\
\text { M-H, Random, } 95 \% \mathrm{Cl}\end{array}$ & & $\begin{array}{r}\text { Odds } \\
M-H, \text { Rand }\end{array}$ & $\begin{array}{l}\text { Ratio } \\
\text { lom, } 95 \% \mathrm{Cl}\end{array}$ & \\
\hline Chen 2011 & 12 & 42 & 12 & 40 & $19.3 \%$ & $0.93[0.36,2.42]$ & & & & \\
\hline Ding 2010 & 2 & 15 & 3 & 15 & $7.5 \%$ & $0.62[0.09,4.34]$ & & & & \\
\hline Duan 2007 & 1 & 12 & 6 & 12 & $5.6 \%$ & $0.09[0.01,0.94]$ & & & & \\
\hline Hillock 2007 & 2 & 14 & 3 & 13 & $7.3 \%$ & $0.56[0.08,4.01]$ & & & & \\
\hline Wang 2008 & 3 & 36 & 15 & 32 & $12.6 \%$ & $0.10[0.03,0.41]$ & & & & \\
\hline Xue 2009 & 11 & 25 & 11 & 23 & $16.0 \%$ & $0.86[0.27,2.67]$ & & & & \\
\hline Zhang 2010 & 6 & 43 & 16 & 44 & $17.3 \%$ & $0.28[0.10,0.82]$ & & & & \\
\hline Zhao 2010 & 5 & 30 & 9 & 30 & $14.4 \%$ & $0.47[0.14,1.61]$ & & & & \\
\hline Total $(95 \% \mathrm{Cl})$ & & 217 & & 209 & $100.0 \%$ & $0.42[0.23,0.76]$ & & & & \\
\hline Total events & 42 & & 75 & & & & & & & \\
\hline $\begin{array}{l}\text { Heterogeneity: } \mathrm{Tau}^{2} \\
\text { Test for overall effect }\end{array}$ & $\begin{array}{l}0.24 ; \mathrm{Chi}^{2} \\
Z=2.84(F\end{array}$ & $\begin{array}{l}=10.6 \\
D=0.0\end{array}$ & $\begin{array}{l}6, d f=7 \\
04)\end{array}$ & $(P=0$. & $15) ; 1^{2}=3$ & & $\begin{aligned} 0.01 \\
\mathrm{Fa}\end{aligned}$ & $\begin{array}{l}0.1 \\
\text { avours BNP }\end{array}$ & $\begin{array}{c}1 \\
\text { Favours con }\end{array}$ & $\begin{array}{l}100 \\
\text { trol }\end{array}$ \\
\hline
\end{tabular}

Figure 6. Comparison of major adverse cardiovascular events (MACEs) between brain natriuretic peptide (BNP) group and control group.

pared with the control group during the short-term follow-up (WMD 6.16\%, 95\% CI: $4.24 \%-8.08 \%$ ) as well as the relative long-term follow-up (WMD 5.64\%, 95\%CI: 3.38\%-7.90\%). We also performed a subgroup analysis of BNP studies with different infusion durations. LVEF was increased by $3.97 \%$ in the BNP group with an infusion time less than 72 hours compared with the control group (95\%CI: $2.03 \%-5.97 \%$ ), and $5.71 \%$ in the BNP group with an infusion time over 72 hours
(95\%CI: $2.57 \%-8.85 \%)$.

The pooled results indicated that MACEs in the BNP group were significantly lower than in the control group (OR: 0.42; 95\% CI: $0.23-0.76)$, with no significant heterogeneity $(P$ $\left.=0.15, \mathrm{I}^{2}=34 \%\right)$ (Figure 6$)$. Nine studies mentioned renal failure incidence, and no patients receiving BNP developed renal failure. Eight studies reported hypotension occurrences after BNP infusion. Studies with isosorbide dinitrate or nitro- 
glycerin as control found that 3 patients developed hypotension in the BNP groups while there were 9 in the control group; and studies with blank control or physiological saline as control reported 4 patients complicated with hypotension in the BNP group compared with 2 in the control group. In all studies that mentioned renal failure cases, patients in the BNP groups did not exhibit a higher frequency of renal failure than the control group.

Considering the Jadad scores of some BNP groups were low, we performed subgroup analysis of studies with a Jadad score above 3 . The results were consistent with the total group analysis (WMD 4.32\%, 95\%CI: $1.62 \%-7.01 \%, P<0.0001, \mathrm{I}^{2}$ $=91 \%$ ). Although most of the studies included in the BNP analysis were performed in China, quality assessment was strictly performed using the Jadad score, which made the results reliable. In addition, we further assessed publication bias using Egger's and Begg's tests for the BNP group, which showed that no potential publication bias existed among the included trials (Egger's test: $P=0.493$; Begg's test: $P=0.893$ ). Because of the statistical heterogeneity across the BNP studies, meta-regression analyses were performed to appraise the impact of different covariates on the changes in LVEF associated with BNP treatment. Specifically, we did not find a statistically significant association between the benefits of BNP treatment and year of publication $(P=0.934)$, patient age $(P=0.883)$, patient gender $(P=0.649)$, baseline LVEF $(P=0.924)$, AHF complication $(P=0.495)$, and infusion duration $(P=0.822)$.

\section{Discussion}

Experimental data have shown that administration of BNP could limit myocardial infarct size in ischemia-reperfusion injured rats through several mechanisms (nitric oxide synthase, ATP-sensitive potassium channel opening, etc), which could reduce the possibility of developing heart failure due to myocardial infarction. ${ }^{4,5)}$ The process of adverse left ventricular remodeling may be initiated soon after AMI, in which the renin-angiotensin-aldosterone system (RAAS) and endothelin play important roles. ANP and BNP have extensive physiological effects including diuresis, natriureis, antifibrosis, antihypertrophy, anti-inflammation and inhibition of sympathetic nerve activity, RAAS, and endothelin synthesis. ${ }^{28-30)}$ Thus, intravenous administration of ANP or BNP is a promising candidate for an adjunctive therapy for AMI, because it might have effects on limiting myocardial infarct size and suppressing the cardiac remodeling process.

We performed meta-analysis of 4 RCTs on ANP and 16 RCTs on BNP to evaluate their therapeutic effect on LVEF improvements in AMI patients. The results indicated that no matter whether AMI patients were complicated with AHF or not; natriuretic peptide infusion could improve LVEF as compared with control. The result of subgroup analysis of different BNP durations showed that longer BNP infusion might generate a stronger effect on ventricular function improvement. As studies in the long-term BNP infusion subgroup mostly maintained BNP infusion for 72 hours, it might be reasonable for AMI patients to receive a 72-hour constant BNP infusion. However, such a larger benefit was not observed in the studies with ANP infusion over 72 hours according to the subgroup analysis results. Furthermore, the recommended maintaining duration for
ANP in the prescribing information was 48 hours. Therefore, it might be sufficient to maintain infusion for 48 hours when ANP was used.

The average LVEF was $2.94 \%$ higher in the ANP groups than in the control during follow-up, while it was $4.45 \%$ higher in the BNP groups compared to the control. It seems that BNP infusion had a better effect on improving LVEF compared with ANP infusion although both types of natriuretic peptides targeted the same receptors. While currently there are no definite explanations for this result, it should be noted that 7 BNP studies included AMI patients complicated with AHF, who might benefit more from BNP infusion as their primary mean LVEF was obviously lower than those in the ANP groups. The recommended dose of recombinant ANP in the prescribing information is intravenous infusion of $0.1 \mu \mathrm{g} / \mathrm{kg} /$ minute (carperitide, Japan), while the recommended dose of recombinant BNP is an intravenous bolus of $1.5-2 \mu \mathrm{g} / \mathrm{kg}$ followed by a continuous infusion of $0.0075-0.01 \mu \mathrm{g} / \mathrm{kg} /$ minute (Xinhuosu, China), and an intravenous bolus of $2 \mu \mathrm{g} / \mathrm{kg}$ followed by a continuous infusion of $0.01 \mu \mathrm{g} / \mathrm{kg} /$ minute (nesiritide, USA). The dose of recombinant ANP used in the relevant studies of the meta-analysis was mainly $0.025 \mu \mathrm{g} / \mathrm{kg} /$ minute, which was lower than the recommended dose; while the dose of recombinant BNP used in the relevant studies of the meta-analysis was mostly the same as the recommended dose. This might also have induced the difference between the ANP and BNP infusion effect on LVEF improvement in AMI patients. The study by Chen, et al also showed that a higher dose of BNP infusion could generate a stronger impact on LVEF improvement, ${ }^{10)}$ but large-scale clinical trials are needed to verify the dose-dependent effect of BNP infusion on AMI patients.

Although an infarct size comparison with maximum CK level or CK-MB level as markers did not exhibit significant differences, a trend toward lower CK or CK-MB peak was seen in the natriuretic peptide infusion arm rather than in the control arm. In addition, the pooled results showed that the incidence of MACEs in the BNP group was significantly lower than in the control group. Pooled analyses based on small samples have suggested that BNP, when compared with placebo, was associated with worsening renal function. ${ }^{31)}$ However, a recent large randomized study on patients with acute decompensated heart failure showed that although BNP infusion was not associated with a decrease in the rate of death and rehospitalization, it was also not related to the worsening of renal function. ${ }^{32)}$ There were no reports of higher occurrence of renal failure in patients receiving ANP/BNP infusion in all the studies included in this article. In the study by O'Connor, et al, BNP infusion was connected to an increase in the rate of hypotension. ${ }^{32)}$ In this article, we were unable to perform a metaanalysis on hypotension by BNP infusion in the setting of AMI because the sample size of each study was relatively small, and the incidence of hypotension was low. However, we could still determine that the incidence of hypotension in the ANP/BNP group was lower than in the group with traditional vasodilator controls such as isosorbide dinitrate and nitroglycerin, but higher than the blank control or physiological saline. In addition, the association between ANP/BNP dose and hypotension occurrence needs further investigation. As AMI patients often suffer from pump failure, ANP/BNP infusion in AMI patients may require special caution with respect to hypotension. Considering ANP has a shorter half-life (2 minutes) than BNP (ap- 
proximately 18 minutes), it may be more appropriate to use ANP when treating AMI patients since it is eliminated more rapidly.

There was significant heterogeneity in the pooled analysis of BNP treatment in AMI patients, so we performed meta-regression analyses to explore potential sources. However, the results showed that publication year, mean age, gender, baseline LVEF, infusion duration, and AHF complications as well as follow-up time were not significantly associated with LVEF improvement. A possible cause of heterogeneity was the different dose in each study. Another possible contributor to the heterogeneity might be the different starting times of BNP infusion. All of the studies included only mentioned the drug was given at the acute phase of AMI, but none of them presented data on the average interval between the onset of AMI and start of infusion. Currently, recombinant ANP (Carperitide, Japan) for patients with heart failure is mainly used in Japan, while in the United States and China, the recombinant form of BNP (Nesiritide, USA; Xinhuosu, China) was used. This explained why in our article the clinical evidence for ANP in treating acute myocardial infarction was obtained in Japan, whereas data on BNP is mainly from the United States and China.

Limitations: Although the findings from this meta-analysis were highly suggestive, we still cannot definitively conclude that additional use of ANP/BNP would not induce higher occurrences of hypotension and renal function deterioration in patients with AMI. Furthermore, the best duration and dosage of ANP/BNP infusion for AMI patients have still not been exactly ascertained. Finally, we did not compare the effects of ANP and BNP in treating AMI.

Conclusion: This meta-analysis showed that ANP/BNP treatment might improve LVEF and reduce the risk of MACEs in AMI patients. Although we were unable to conclude whether ANP/BNP infusion could cause renal function deterioration and hypotension, among the studies included in this metaanalysis, we did not see a higher incidence of renal function failure and hypotension in the ANP/BNP groups than in the control groups. Therefore, ANP/BNP infusion may be a potential novel adjunctive treatment strategy for AMI patients.

\section{REFERENCES}

1. Bettencourt P. Brain natriuretic peptide (nesiritide) in the treatment of heart failure. Cardiovasc Drug Rev 2002; 20: 27-36. (Review)

2. Saito Y. Roles of atrial natriuretic peptide and its therapeutic use. J Cardiol 2010; 56: 262-70. (Review)

3. Richards AM. Therapeutic potential of infused cardiac natriuretic peptides in myocardial infarction. Heart 2009; 95: 1299-300.

4. D'Souza SP, Yellon DM, Martin C, et al. B-type natriuretic peptide limits infarct size in rat isolated hearts via KATP channel opening. Am J Physiol 2003; 284: H1592-600.

5. Ren B, Shen Y, Shao H, Qian J, Wu H, Jing H. Brain natriuretic peptide limits myocardial infarct size dependent of nitric oxide synthase in rats. Clin Chim Acta 2007; 377: 83-7.

6. Takata Y, Hirayama Y, Kiyomi S, et al. The beneficial effects of atrial natriuretic peptide on arrhythmias and myocardial high-energy phosphates after reperfusion. Cardiovasc Res 1996; 32: 28693.

7. Jadad AR, Moore RA, Carroll D, et al. Assessing the quality of reports of randomized clinical trials: is blinding necessary? Control Clin Trials 1996; 17: 1-12.
8. Cai WD, Li XL, Bi YP. Effectiveness and safety of recombinant human brain natriuretic peptide in aged patients with acute myocardial infarction complicated with heart failure. Acta Academiae Med Shandong 2010: 92-4.

9. Chen D, Huang L, Shang YJ. Effects of intravenous recombinant human brain natriuretic peptide after primary PCI on ventricular remodeling and ventricular function in patients with acte myocardial infarction. Jilin Med J 2011: 11-3.

10. Chen HH, Martin FL, Gibbons RJ, et al. Low-dose nesiritide in human anterior myocardial infarction suppresses aldosterone and preserves ventricular function and structure: a proof of concept study. Heart 2009; 95: 1315-9.

11. Ding ZJ. The short-term observation of rhBNP infusion in AMI patients receiving primary PCI [Master's paper]: Dalian Medical University; 2008.

12. Duan JB, Fu XH. The effect of intravenous infusion of rhBNP on left ventricular remodeling in AMI patients receiving XXX???? [Master's paper]: Hebei Medical University; 2007.

13. Hayashi M, Tsutamoto $\mathrm{T}$, Wada A, et al. Intravenous atrial natriuretic peptide prevents left ventricular remodeling in patients with first anterior acute myocardial infarction. J Am Coll Cardiol 2001; 37: 1820-6.

14. Hillock RJ, Frampton CM, Yandle TG, Troughton RW, Lainchbury JG, Richards AM. B-type natriuretic peptide infusions in acute myocardial infarction. Heart 2008; 94: 617-22.

15. Kasama S, Toyama T, Hatori $\mathrm{T}$, et al. Effects of intravenous atrial natriuretic peptide on cardiac sympathetic nerve activity and left ventricular remodeling in patients with first anterior acute myocardial infarction. J Am Coll Cardiol 2007; 49: 667-74.

16. Kitakaze M, Asakura M, Kim J, et al. Human atrial natriuretic peptide and nicorandil as adjuncts to reperfusion treatment for acute myocardial infarction (J-WIND): two randomised trials. Lancet 2007; 370: 1483-93.

17. Kuga H, Ogawa K, Oida A, et al. Administration of atrial natriuretic peptide attenuates reperfusion phenomena and preserves left ventricular regional wall motion after direct coronary angioplasty for acute myocardial infarction. Circ J 2003; 67: 443-8.

18. Li HB, Xin JM, Zhao LC, Zhang Y, Sun WH, Zhu WB. Clinical evaluation of early low-dose nesiritide injection relieves left ventricular remodeling in acute myocardial infarction patients after PCI. Jilin Med J 2011: 690-2. (Chinese)

19. Liu CH, Lu JK, Li YH, Wang FZ. The function of BNP in acute myocardial infarction patients with heart failure. Heilongjiang Med Pharm 2010: 63-4. (Chinese)

20. Liu ND, Zhou X. Effect of rhBNP infusion in AMI patients complicated with acute heart failure. J Chinese Biochem 2011: 23940.

21. Long SQ, Liu B, Zou GH, Chen YH. Effect of rhBNP therapy in AMI patients complicated with heart failure. J Chinese Med Drugs 2011: 81-2.

22. Ma YY, Han ZL, Wang SL, Wang DM. Clinical evaluation of rhBNP function in acute pump failure after STEMI. South China J Cardiovasc Dis 2009; Special Edition: 64.

23. Wang DY, Jin HG. Efficiency and safety evaluation of natriuretic peptide in treating acute myocardial infarction complicated with heart failure. J Shanghai Yixue 2008: 411-3.

24. Xue L, Fu XH, Wu WL, et al. rhBNP infusion on ventricular remodeling and contraction synchronism in AMI patients after PCI. J Chinese Circulation 2009: 170-3.

25. Zhang J, Fu XH, Jia XW. Efficacy and safety of recombinant human B-type natriuretic peptide on acute systolic heart failure patients with acute anterior myocardial infarction. China J Gereol 2010; 30: 438-41.

26. Zhao Q, Wu TG, Lin Y, Li B, Luo JY, Wang LX. Low-dose nesiritide improves renal function in heart failure patients following acute myocardial infarction. Heart and Vessels 2010; 25: 97-103.

27. Zou RX, Xu ZP, Ding ZJ. Clinical evaluation of using BNP to treat acute myocardial infarction patients with heart failure. Med Innovation Res 2007; 4: 114.

28. Nishikimi T, Maeda N, Matsuoka H. The role of natriuretic pep- 
tides in cardioprotection. Cardiovasc Res 2006; 69: 318-28. (Review)

29. Silver MA. The natriuretic peptide system: kidney and cardiovascular effects. Curr Opin Nephrol Hypertens 2006; 15: 14-21. (Review)

30. Woods RL. Cardioprotective functions of atrial natriuretic peptide and B-type natriuretic peptide: a brief review. Clin Exp Pharmacol
Physiol 2004; 31: 791-4. (Review)

31. Sackner-Bernstein JD, Skopicki HA, Aaronson KD. Risk of worsening renal function with nesiritide in patients with acutely decompensated heart failure. Circulation 2005; 111: 1487-91.

32. O'Connor CM, Starling RC, Hernandez AF, et al. Effect of nesiritide in patients with acute decompensated heart failure. $\mathrm{N}$ Engl $\mathrm{J}$ Med 2011; 365: 32-43. 